\title{
TOIT Reviewers over 2017
}

TOIT's success in maintaining its standards of quality and timeliness relies in large part on the efforts of peer reviewers. Since the review assignments for any article or issue are kept anonymous to promote impartiality, we acknowledge their efforts en masse here. This list includes all those who submitted at least one review from January 1, 2017 to December 31, 2017.

On behalf of TOIT authors, associate editors, and guest editors, I thank you for your professional service.

Munindar P. Singh

Editor-in-Chief

1. Adamatti, Diana

2. Adjerid, Idris

3. Afifi, Hossam

4. Al Hasan, Mohammad

5. Aleti, Aldeida

6. Allahbakhsh, Mohammad

7. An, Bo

8. Andreou, Athanasios

9. Anvik, John

10. Ardagna, Claudio

11. Armas Cervantes, Abel

12. Artikis, Alexander

13. Aydogan, Reyhan

14. Azzini, Antonia

15. Bagheri, Ebrahim

16. bajpai, vaibhav

17. Bandara, Dilum

18. Bardas, Alexandru

19. Baroglio, Cristina

20. Beckers, Kristian

21. Beheshti, Amin

22. Belk, Marios

23. Bellandi, Valerio

24. Benatallah, Boualem

25. Benton, Ryan

26. Bhardwaj, Onkar

27. Bhiri, Sami

28. Bielova, Nataliia

29. Biener, Christian

30. Bimpikis, Kostas
31. Bird, Christian

32. Böhme, Rainer

33. Boissier, Olivier

34. Bond, Mike

35. Bonneau, Joseph

36. Bonnet, Gregory

37. Bork, Dominik

38. Botev, Jean

39. Bouassida, Ismail

40. Bouzefrane, Samia

41. Brafman, Ronen

42. Branch, Philip

43. Brandimarte, Laura

44. Breitenbuecher, Uwe

45. Broersen, Jan

46. Brogi, Antonio

47. Buntain, Cody

48. Burnap, Pete

49. Cai, Hongming

50. Cánovas, Javier

51. Cao, Yinzhi

52. Catalini, Christian

53. Chabridon, Sophie

54. Chan, Hau

55. Chang, Chii

56. Charoy, Francois

57. Chau, Sze Yiu

58. Chen, Ang

59. Chen, Bolun

60. Chen, Dong

2018 Copyright is held by the owner/author(s).

1533-5399/2018/11-ART57

https://doi.org/10.1145/3232919 


\author{
61. Chen, Haining \\ 62. Chen, Shuang \\ 63. Cherrier, Sylvain \\ 64. Chessa, Michela \\ 65. Chew, Eng \\ 66. Chi, Chi-Hung \\ 67. Chirkova, Rada \\ 68. Cho, Jin-Hee \\ 69. Chowdhury, Omar \\ 70. Christin, Nicolas \\ 71. Cimato, Stelvio \\ 72. Clayman, Stuart \\ 73. Clayton, Richard \\ 74. Cong, Gao \\ 75. Costa, Fabio \\ 76. Costin, Andrei \\ 77. Courcoubetis, Costas \\ 78. Cremonini, Marco \\ 79. Criado, Natalia \\ 80. Cubo, Javier \\ 81. Cui, Tiansong \\ 82. Dalpiaz, Fabiano \\ 83. Dam, Hoa \\ 84. Damiani, Prof. Ernesto \\ 85. Daniel, Florian \\ 86. Datta, Amit \\ 87. Day, Wei-Yen \\ 88. delicato, flavia \\ 89. del-Río-Ortega, Adela \\ 90. Demartini, Gianluca \\ 91. Dennis, Louise \\ 92. Desai, Nirmit \\ 93. Dhamal, Swapnil \\ 94. Diccicio, Claudio \\ 95. Dijkman, Remco \\ 96. Dong, Hai \\ 97. Dou, Wanchun \\ 98. Doupé, Adam \\ 99. Duan, Yucong \\ 100. Duggimpudi, Maria B \\ 101. Dumas, Marlon \\ 102. Eksin, Ceyhun \\ 103. Elmokashfi, ahmed \\ 104. Engel, Robert \\ 105. Eshuis, Rik \\ 106. Fang, Yong
}

107. Farhang, Sadegh

108. Fdhila, Walid

109. Ferry, Nicolas

110. Figueiredo, Flavio

111. Filiposka, Sonja

112. Fischer-Hübner, Simone

113. Forné, Jordi

114. Fortino, Giancarlo

115. Gañan, Carlos

116. Gangadharan, G R

117. Garcia-Fornes, Ana

118. Garciia-Valls, Marisol

119. Geambasu, Roxana

120. Gerlach, Jin

121. Ghari Neiat, Azadeh

122. Ghose, Aditya

123. Gianini, Gabriele

124. Glavic, Boris

125. Goel, Arpit

126. Gómez Mármol, Félix

127. Gong, Neil

128. Governatori, Guido

129. Graf-Vlachy, Lorenz

130. Graiet, Mohamed

131. Grossi, Davide

132. Grossklags, Jens

133. Grossmann, Georg

134. Gunay, Akin

135. Guo, Linke

136. Gurcan Akcora, Cuneyt

137. Haddadi, Hamed

138. Hafez, Ahmed Ibrahem

139. Hang, Chung-Wei

140. Haniewicz, Konstanty

141. Hankin, Chris

142. HARPER, Simon

143. Hashmi, Mustafa

144. He, Xiaofan

145. He, Xinean

146. Hegde, Aditya

147. Hegde, Nidhi

148. Herley, Cormac

149. Hill, James

150. Hirsch, Benjamin

151. Hochreiner, Christoph

152. Horne, Ben 
153. Horng, Shi-Jinn

154. Hossfeld, Tobias

155. Hsu, Hwai-Jung

156. Hu, Hongxin

157. Huber, Jochen

158. Hübner, Jomi

159. Huguenin, Kevin

160. Hunter, Anthony

161. Imran, Muhammad

162. Ioannidis, Stratis

163. Ishakian, Vatche

164. Jain, Rakesh

165. Javed, Mobin

166. Jia, Yue

167. Jiao, Lei

168. Jimenez-Ruiz, Ernesto

169. Jones, Christopher

170. Jones, Simon

171. Jorgensen, Zach

172. Kafali, Ozgur

173. Kalia, Anup

174. Kallel, Slim

175. Kamble, Vijay

176. Karastayanova, Dimka

177. Katzenbeisser, Stefan

178. Kennedy, Oliver

179. Khan, Asjad

180. Khan, Khaled M.

181. Khouzani, MHR.

182. King, Thomas

183. Klinkmuller, Christopher

184. Koetter, Falko

185. Köhler, Olaf Markus

186. Kohout, Jan

187. Koll, David

188. Kollingbaum, Martin

189. Köpf, Boris

190. Koppel, Ross

191. Kounev, Samuel

192. Kowalczyk, Ryszard

193. Kratzke, Nane

194. Ksihnamurthy, Balachander

195. Kuflik, Tsvika

196. Lai, Chin-Hui

197. Laszka, Aron

198. Laube, Stefan
199. Laurie, Ben

200. Lecuyer, Mathias

201. Lee, Ickjai

202. Lee, Joyce Yi-Hui

203. Leitner, Philipp

204. Lemmerich, Florian

205. Leneutre, Jean

206. Leverett, Eireann

207. Li, Xianxian

208. Li, Ze

209. Lima, Ana C. E. S.

210. Lin, Jianyi

211. Lin, Shian-Hua

212. Lin, Xue

213. Lisy, Viliam

214. Liu, Shenghua

215. Liu, Wenyin

216. Liu, Xuanzhe

217. Loeb, Marty

218. Loiseau, Patrick

219. Luckie, Matthew

220. Luhn, Sebastian

221. Lui, John C.S.

222. Lumpe, Marcus

223. Lyu, Michael

224. Maamar, Zakaria

225. Malawski, Maciej

226. Mammar, Amel

227. Mandal, Amit Kr

228. Manshaei, Mohammad

229. Marin-Castro, Heidy M.

230. Marotta, Veronica

231. Mascardi, Viviana

232. Maurice, Clementine

233. Mayer, Jonathan

234. McClean, Sally

235. Meneguzzi, Felipe

236. Merle, Dr. Philippe

237. Mhamed, Abdallah

238. Miles, Simon

239. Misic, Vojislav

240. Misra, Gaurav

241. Mistry, Sajib

242. Mital, Pratik

243. Moeser, Malte

244. Mohamed, Mohamed 
245. Moisan, Frederic

246. Mondal, Mainack

247. Moore, Tyler

248. Moreno, Antonio

249. Morlok, Tina

250. Motahari, Hamid

251. Muhlenbach, Fabrice

252. Murdoch, Steven

253. Murukannaiah, Pradeep

254. Nagappan, Meiyappan

255. Nahorski, Zbigniew

256. Narang, Pratik

257. Nehring, Klaus

258. Nolle, Timo

259. Ögüt, Hulisi

260. Onaindia, Eva

261. oya, simon

262. Padget, Julian

263. Pahl, Claus

264. Pal, Ranjan

265. Palanisamy, Balaji

266. Panaousis, Emmanouil

267. Pardo, Raul

268. Patil, Sameer

269. Pellegrino, Giancarlo

270. Peng, Wei

271. Pevny, Tomas

272. Piolle, Guillaume

273. Pitt, Jeremy

274. Polakis, Jason

275. Pozzato, Gian Luca

276. Prakken, Henry

277. Preibusch, Sören

278. Prodan, Radu

279. $\mathrm{Pu}, \mathrm{Yu}$

280. Qiao, $\mathrm{Mu}$

281. Qin, Yongrui

282. Qu, Lie

283. Ranjan, Rajiv

284. Reed, Karl

285. Rehak, Martin

286. Reichert, Manfred

287. Ren, Wei

288. Romanosky, Sasha

289. Rossi, Gian Paolo

290. Rossow, Christian
291. Rovatsos, Michael

292. Ruffo, Giancarlo

293. Saad, Walid

294. Sadiq, Shazia

295. Sahin, Merve

296. Saleh, Iman

297. Salim, Flora

298. SAMAMA, Porf Nel

299. Scafuro, Alessandra

300. Schall, Daniel

301. Schaub, Florian

302. Schifanella, Claudio

303. Schneider, Jean-Guy

304. Schwartz, Galina

305. Scotney, Bryan

306. Secci, Stefano

307. Seinturier, Lionel

308. Sellami, Mohamed

309. Sha, Kewei

310. Shahzad, Muhammad

311. Sheng, Quan Z.

312. Sillaber, Christian

313. Sindhgatta, Renuka

314. Singh, Vivek

315. Sliman, Layth

316. Sloan, Robert

317. Smyth, Ben

318. Song, Guangfeng

319. Song, Xuan

320. Soulie, Nicolas

321. Spahn, Riley

322. Spiekermann, Sarah

323. squicciarini, anna

324. Staples, Mark

325. Stringhini, Gianluca

326. Su, Dong

327. Sun, Hailong

328. Taconet, Chantal

329. Taheri, Javid

330. Tang, Yuzhe

331. Tata, Samir

332. Tavanaei, Amirhossein

333. Telang, Rahul

334. Terzi, Evimaria

335. Tessier, Catherine

336. Teyeb, Hana 
337. Theisen, Chris

338. Theodorakopoulos, George

339. Thielmann, Isabel

340. Tilevich, Eli

341. Tippenhauer, Nils

342. Tkalcic, Marko

343. Tolosana-Calasanz, Rafael

344. Tonetta, Stefano

345. Toumani, Farouk

346. Traverso, Stefano

347. Truong, Hong-Linh

348. Tschantz, Michael Carl

349. Tuffin, Bruno

350. Unland, Rainer

351. Ur, Blase

352. Van Hoorn, André

353. van Wieren, Maarten

354. Varghese, Blesson

355. Venkatadri, Giridhari

356. Vetterl, Alexander

357. Vizzari, Giuseppe

358. Vo, Bao

359. Vucovic, Maja

360. Wagman, Liad

361. Wang, Gang

362. Wang, Lingyu

363. Wang, Qia

364. Wang, Xiaoyin

365. Warnier, Martijn

366. Weber, Dr Ingo

367. Weber, Steven

368. Weichbroth, Pawel
369. Weidlich, Matthias

370. Wilson, Christo

371. Wolter, Katinka

372. Wu, Fan

373. Wu, Ou

374. Wu, Zhiang

375. Xiang, Yang

376. Xie, Tao

377. Xie, Ying

378. Xu, Feng

379. Yang, Ye

380. Yangui, Sami

381. Yao, Xuanxia

382. Yeob Yeun, Chan

383. Yigitoglu, Emre

384. Yin, Peifeng

385. Yolum, Pinar

386. Yu, Qi

387. Yue, Tao

388. Zannone, Nicola

389. Zeng, Qingtian

390. Zhang, Hao

391. Zhang, Xiang-Liang

392. Zhang, Zhe

393. Zhao, Xiaohui

394. Zheleva, Elena

395. Zheng, Kan

396. Zheng, Xi

397. Zheng, Xiaolin

398. Zhong, Ning

399. Zhou, Bin

400. Zhu, Yihai 